\title{
MicroRNA-325-3p contributes to colorectal carcinoma by targeting cytokeratin 18
}

\author{
CHUANFANG SONG, XIUJIE WANG, XINXIN ZHAO, JIANG AI, YIXUAN QI and AIDONG CHEN \\ Department of Gastroenterology, Hongqi Hospital Affiliated to Mudanjiang Medical University, \\ Mudanjiang, Heilongjiang 157011, P.R. China
}

Received August 31, 2020; Accepted December 23, 2020

DOI: $10.3892 / 01.2021 .12509$

\begin{abstract}
Colorectal carcinoma (CRC) is one of the most common malignant tumors. The present study aimed to investigate a non-invasive molecular marker that can evaluate the diagnosis and potential molecular mechanism of CRC. Microarray assays and reverse transcription-quantitative PCR analysis demonstrated that microRNA (miR)-325-3p expression was significantly increased in both tissues and serum samples of patients with CRC. In addition, miR-325-3p expression in the tissues and serum was significantly associated with differentiation, TNM stage and lymph node metastasis. The results of the dual-luciferase reporter assay and western blot analysis revealed that cytokeratin 18 (CK18) is a target gene of miR-325-3p. Furthermore, treatment with transforming growth factor (TGF)- $\beta$ increased miR-325-3p expression in a time-dependent manner. Conversely, TGF- $\beta$ decreased CK18 expression at 48 and $72 \mathrm{~h}$. Western blot analysis demonstrated that TGF- $\beta 1$ significantly decreased the expression of the epithelial marker, CK18, and increased the expression of the mesenchymal markers, $\alpha$-SMA and vimentin. Notably, these effects were reversed following inhibition of miR-325-3p expression. Taken together, the results of the present study suggest that miR-325-3p is a key regulator of TGF- $\beta$-induced CK18 downregulation. Thus, elevated levels of miR-325-3p is an important factor affecting epithelial-to-mesenchymal transition, and is likely to be a molecular marker in the progression of CRC and act as a potential therapeutic target.
\end{abstract}

\section{Introduction}

Colorectal carcinoma (CRC) is one of the most common malignant tumors, with the incidence of CRC being 15-25/100,000 population in China in $2011(1,2)$. Given that there are no

Correspondence to: Dr Aidong Chen, Department of Gastroenterology, Hongqi Hospital Affiliated to Mudanjiang Medical University, 5 Tongxiang Road, Mudanjiang, Heilongjiang 157011, P.R. China

E-mail: xiaohua202009@163.com

Key words: microRNA-325-3p, colorectal carcinoma, cytokeratin 18 specific symptoms in the early stage of CRC, patients are diagnosed when the tumor is in the advanced stage (3). Despite surgical treatment, the 5-year overall survival (OS) rate is still $<50 \%$ (3). Thus, one of the most effective ways to improve the survival rate is to identify molecular markers that can reflect the invasion, metastasis and prognosis of CRC, and provide targeted adjuvant treatment to patients $(4,5)$.

Recently, it has been demonstrated that small non-coding microRNAs (miRNAs/miRs) play an important role in tumor-related gene regulation $(6,7)$. miRNAs are highly conserved, single-stranded non-coding small RNA molecules $(8,9)$. As endogenous regulators, they regulate the expression of target genes by binding to the 3'-untranslated region (UTR) of target mRNAs at the post-transcriptional level (10). Increasing evidence suggests that abnormal expression of miRNAs is associated with the occurrence, invasion and metastasis of human malignant tumors, indicating that miRNAs are involved in the regulation of tumorigenesis, invasion and metastasis (11-13). For example, miR-92b-3p contributes to CRC invasion by inhibiting FBXW7 (9). In addition, increased levels of miR-15a-5p predict poor disease-free survival and OS of patients with CRC (14).

The present study screened the miRNAs that are dysregulated in the tissues and serum samples of patients with CRC via microarray assay. The present study aimed to investigate miRNAs in CRC and target genes that are associated with CRC.

\section{Materials and methods}

Patient samples. A total of 50 patients with CRC (male/female ratio, 24/26; mean age $\pm S D, 53.6 \pm 15.8$ years; age range, $34-73$ years) and 21 healthy individuals (male/female ratio, $11 / 10$; mean age \pm SD, 52.8 \pm 17.4 years; age range, $36-72$ years) were recruited in the present study between January 2019 and December 2019. Due to financial constraints, the present study could not recruit equal numbers of healthy controls and patients with CRC. Among the patients with CRC, 46 patients received radical surgery, while 4 patients did not receive surgery. CRC tissues and adjacent non-cancer tissues $(\geq 5 \mathrm{~cm})$ were collected. The patient characteristics are present in Table I. All patients had complete clinical and pathological data, and the inclusion criteria were as follows: i) CRC was diagnosed via histopathology at the initial diagnosis and 
treatment; ii) no radiotherapy or chemotherapy was received prior to treatment; iii) no significant abnormalities of heart, liver or kidney functions were observed and iv) no other tumor or serious diseases were observed. Peripheral blood $(5 \mathrm{ml})$ was collected from all patients with CRC on an empty stomach following surgery, and $5 \mathrm{ml}$ of peripheral blood was collected from the healthy individuals. The samples were centrifuged at $3,500 \mathrm{x} \mathrm{g}$ for $15 \mathrm{~min}$ at $4^{\circ} \mathrm{C}$ and the supernatant was stored at $-80^{\circ} \mathrm{C}$ until subsequent experimentation. The present study was approved by the Medical Ethics Committee of Hongqi Hospital Affiliated to Mudanjiang Medical University (Mudanjiang, China; approval no. MDJHQ-20180925), and written informed consent was provided by all participants prior to the study start.

RNA isolation. Total RNA was extracted from the tissues and serum samples using RNAVzol (Vigorous Biotechnology Beijing Co., Ltd.), according to the manufacturer's protocol. The concentration and purity of the RNA samples were determined by measuring the optical density (OD) 260/OD 280.

Microarray assay. To compare the miRNA transcriptome between CRC tissues/serum samples and the respective controls, seven different samples were taken as one mixture. For each group, three different mixtures were included in each group for miRNA profiling using an Agilent miRNA array (Agilent Technologies, Inc.). Each miRNA was detected using probes (Agilent Technologies, Inc.) and repeated 30 times. The array also contained 2,164 Agilent control probes. The miRNA samples were Cy3 labeled using the Agilent miRNA Complete Labeling and Hyb kit (cat. no. 5190-0456; Agilent Technologies, Inc.), according to the manufacturer's instructions. The differentially expressed miRNAs were obtained using a combine threshold of fold change $>1.5$ and t-test $\mathrm{P}<0.05$ for transcriptome comparisons.

Reverse transcription-quantitative $(R T-q) P C R$. Total RNA was isolated from the serum and tissue samples using RNAVzol LS or RNAVzol (Vigorous Biotechnology Beijing Co., Ltd.) according to the manufacturer's protocol. The concentration and the purity of RNA samples was determined by measuring the optical density (OD) 260/OD280. A total of $1 \mu \mathrm{g}$ RNA was reverse transcribed using Moloney Murine Leukemia Virus (MMLV) reverse transcription enzyme (Applied Biosystems; Thermo Fisher Scientific, Inc.) with specific primers. The RT system $(10 \mu \mathrm{l})$ was composed as follows: $3.5 \mu \mathrm{l}$ of DEPC water, $2.0 \mu \mathrm{l}$ of $5 \mathrm{X}$ reverse transcription buffer, $1.0 \mu \mathrm{l}$ of $10 \mathrm{mmol} / \mathrm{l}$ dNTPs, $0.5 \mu \mathrm{l}$ of MMLV reverse transcriptase (all Applied Biosystems; Thermo Fisher Scientific, Inc.), $1.0 \mu 1$ of miRNA reverse transcription primer and $2.0 \mu \mathrm{l}$ of RNA sample. The reaction conditions were as follows: $16^{\circ} \mathrm{C}$ for $30 \mathrm{~min}, 42^{\circ} \mathrm{C}$ for $30 \mathrm{~min}, 85^{\circ} \mathrm{C}$ for $5 \mathrm{~min}$ and hold at $4^{\circ} \mathrm{C}$. To quantify the relative mRNA levels, qPCR was performed using SYBR Green Supermix (Bio-Rad Laboratories, Inc.) in an iCycleriQ real-time PCR detection system. The reaction conditions were as follows: $95^{\circ} \mathrm{C}$ for $5 \mathrm{~min}$, followed by 40 cycles of $95^{\circ} \mathrm{C}$ for $15 \mathrm{sec}$ and $60^{\circ} \mathrm{C}$ for $1 \mathrm{~min}$. Relative miRNA expression was calculated using the $2^{-\Delta \Delta C q}$ method (15) and normalized to the internal reference gene U6. The primers used in the present study were as follows: miR-325-3p-RT, 5'-GTCGTATCCAGT
GCAGGGTCCGAGGTATTCGCACTGGATACGACAAAT AAC-3'; U6-RT, 5'-GTCGTATCCAGTGCAGGGTCCGAG GTATTCGCACTGGATACGACAAAATG-3'; miR-325-3p forward, 5'-GCGCAACTATCCTCCAGG-3'; U6 forward, 5'-GCGCGTCGTGAAGCGTTC-3'; universal reverse primer for miR-325-3p and U6, 5'-GTGCAGGGTCCGAGGT-3'.

Cell culture. The human CRC cell line, HT-29, was purchased from the American Type Culture Collection and authenticated via STR profiling. The cells were maintained in Ham's F-12 nutrient medium (Invitrogen; Thermo Fisher Scientific, Inc.) supplemented with $10 \%$ fetal bovine serum (FBS; HyClone; Cytiva), $100 \mathrm{U} / \mathrm{ml}$ penicillin (Invitrogen; Thermo Fisher Scientific, Inc.) and $0.1 \mathrm{mg} / \mathrm{ml}$ streptomycin (HyClone; Cytiva), at $37^{\circ} \mathrm{C}$ with $5 \% \mathrm{CO}_{2}$.

The human CRC cell lines, SW480 and HCT116, the normal colon cell line, FHC, and 293T cells were purchased from the Chinese Academy of Sciences Cell Bank of Type Culture Collection. All cells were maintained in RPMI-1640 medium (HyClone; Cytiva) supplemented with $10 \% \mathrm{FBS}$, $100 \mathrm{U} / \mathrm{ml}$ penicillin and $100 \mathrm{mg} / \mathrm{ml}$ streptomycin, at $37^{\circ} \mathrm{C}$ with $5 \% \mathrm{CO}_{2}$.

Transient transfection. miR-325-3p mimic (5'-AACTATCCT CCAGGAGTTATTT-3') or inhibitor (5'-AAATAACTCCTG GAGGATAGTT-3') and the respective negative controls (NCs; NC mimic, 5'-TTCTCCGAACGTGTCACGT-3'; NC inhibitor, 5'-TTCTCCGAACGTTGTCACGT-3') (all from Shanghai GenePharma Co., Ltd.) are chemically modified analogs, which can be transfected into cells without using a vector. Transfection was performed using HiPerFect Transfection Reagent (Qiagen $\mathrm{GmbH}$ ), as previously described (16). Briefly, HT-29 cells were seeded in a 6 -well plate at a density of $10^{6}$ cells/well. Subsequently, the cells were transfected with miR-325-3p mimic, inhibitor or NC for $48 \mathrm{~h}$ using HiPerFect Transfection Reagent according to the manufacturer's instructions. A total of $12 \mu \mathrm{l}$ HiPerFect Transfection Reagent was mixed with $100 \mu \mathrm{l}$ cell culture in serum-free DMEM (Invitrogen; Thermo Fisher Scientific, Inc.). Meanwhile, $10 \mu \mathrm{l}$ miR-325-3p mimic, inhibitor or NC was mixed with serum-free DMEM. Then, the two mixtures were mixed and incubated at room temperature for $15 \mathrm{~min}$. After that, the mixture was added in the 6 -well plate at a final concentration of $20 \mathrm{nM}$. Following transfection for $48 \mathrm{~h}$, the cells were collected for subsequent experiments.

Cell viability analysis. To examine cell viability, HT-29 cells were seeded in 96-well plates at a density of 1.0x10 4 cells/well. miR-325-3p mimics, inhibitors or NC were transfected into cells and the viability of transfected cells was measured at 24 , 48 and $72 \mathrm{~h}$ after seeding of cells. MTT assay was performed as previously described (17).

Cell migration and invasion. Cell migration assays were performed using Boyden chambers $(8-\mu \mathrm{m}$ pore filter; Corning Inc.). For the cell invasion assay, the filter surfaces were precoated with Matrigel (BD Biosciences) at $37^{\circ} \mathrm{C}$ for $2 \mathrm{~h}$. Briefly, transfected HT-29 cells were seeded at a density of $10^{5}$ cells/well in the upper chamber for $24 \mathrm{~h}$ in RPMI-1640 medium without FBS. RPMI-1640 medium (600 $\mu$ l) with $20 \%$ FBS was plated in the lower chamber. After $48 \mathrm{~h}$ of incubation 
Table I. Clinicopathological characteristics of patients with colorectal cancer $(n=50)$.

\begin{tabular}{|c|c|}
\hline Characteristic & Number of patients, $n$ \\
\hline \multicolumn{2}{|l|}{ Sex } \\
\hline Male & 24 \\
\hline Female & 26 \\
\hline \multicolumn{2}{|l|}{ Age, years } \\
\hline$\geq 60$ & 32 \\
\hline$<60$ & 18 \\
\hline \multicolumn{2}{|l|}{ Location } \\
\hline LSCC & 9 \\
\hline RSCC & 17 \\
\hline Rectum & 24 \\
\hline \multicolumn{2}{|l|}{ Type } \\
\hline Protuberant & 19 \\
\hline Ulcerative & 23 \\
\hline Invasive & 8 \\
\hline \multicolumn{2}{|l|}{ Differentiation } \\
\hline Poor & 16 \\
\hline Poor/Moderate & 14 \\
\hline Moderate & 12 \\
\hline Well & 8 \\
\hline \multicolumn{2}{|l|}{ TNM } \\
\hline $\mathrm{I} / \mathrm{II}$ & 21 \\
\hline III/IV & 29 \\
\hline \multicolumn{2}{|c|}{ Lymph node metastasis } \\
\hline No & 23 \\
\hline Yes & 27 \\
\hline
\end{tabular}

LSCC, lateral semicircular canal; RSCC, right sided colon carcinoma; TNM, tumor-node-metastasis.

at $37^{\circ} \mathrm{C}$, non-migratory and non-invading cells were removed with cotton swabs. The migratory or invasive cells located on the lower side of the chamber were fixed in methanol for $30 \mathrm{~min}$ at $37^{\circ} \mathrm{C}$ and stained with $0.5 \%$ crystal violet for $1 \mathrm{~h}$ at $37^{\circ} \mathrm{C}$. Stained cells were counted in 5 random fields using fluorescence microscopy (magnification, x40). All experiments were performed in triplicate.

Dual-luciferase reporter assay. Based on the TargetScan database (http://www.targetscan.org/mamm_31/), a conserved binding site was identified in the 3'-UTR of cytokeratin 18 (CK18). The 3'-UTR of CK18 containing the predicted binding site was cloned into the pmirGLO luciferase reporter vector (Promega Corporation). Subsequently, the plasmid and/or miR-325-3p mimic was transfected into HT-29 cells using Vigofect transfection reagent (Vigorous Biotechnology Beijing Co., Ltd.), according to the manufacturer's protocol. $293 \mathrm{~T}$ cells were seeded in a 6-well plate at a density of $10^{6}$ cells/well. Subsequently, the cells were transfected with miR-325-3p mimic (5'-AACTATCCTCCAGGAGTTATTT-3') or NC (5'-TTCTCCGAACGTGTCACGT-3') (both Shanghai
GenePharma Co., Ltd.) for 48 h using Vigofect transfection reagent (Vigorous Biotechnology Beijing Co., Ltd.) according to the manufacturer's instructions. Briefly, $10 \mu \mathrm{l}$ Vigofect transfection reagent was mixed with $100 \mu \mathrm{l}$ cell culture in serum-free DMEM. Meanwhile, $10 \mu 1 \mathrm{miR}-325-3 \mathrm{p}$ mimic or NC and pmirGLO-CK18-3'-UTR plasmid was mixed with the aforementioned mixture. Then, the two mixtures were mixed and incubated at room temperature for $10 \mathrm{~min}$. Subsequently, the mixture was added in the 6-well plate at a final concentration of $20 \mathrm{nM}$. Following transfection for $48 \mathrm{~h}$, the luciferase activity was detected using a Dual Luciferase Reporter Assay System (Promega Corporation). Firefly luciferase activity was normalized to Renilla luciferase activity.

Western blotting. Total protein isolated from CRC samples or HT-29 cells was extracted using RIPA buffer (Beijing Solarbio Science \& Technology Co., Ltd.). A bicinchoninic protein assay kit (Pierce; Thermo Fisher Scientific, Inc.) was used to determine the protein concentration. A total of $20 \mu \mathrm{g}$ protein/lane was separated by $10 \%$ SDS-PAGE and subsequently transferred onto PVDF membranes (EMD Millipore). Membranes were blocked with $5 \%$ skimmed milk at room temperature for $2 \mathrm{~h}$. The membranes were incubated with primary antibodies against: CK18 (1:1,000; cat. no. ab133263; Abcam), $\alpha$-SMA (1:1,000; cat. no. 19245; Cell Signaling Technology, Inc.), vimentin (1:1,000; cat. no. 5741; Cell Signaling Technology, Inc.) and GAPDH (1:1,000; cat. no. 5174; Cell Signaling Technology, Inc.) overnight at $4^{\circ} \mathrm{C}$. Membranes were washed with PBST three times, and subsequently incubated with HRP-conjugated anti-rabbit secondary antibodies (1:5,000; cat. no. ZB-2301; Beijing Zhongshan Golden Bridge Biotechnology Co., Ltd.) for $2 \mathrm{~h}$ at room temperature. Protein bands were visualized using the ECL Plus detection system (EMD Millipore), according to the manufacturer's instructions. GAPDH was used as the internal control.

TGF- $\beta$ treatment. HT-29 cells were treated with $10 \mathrm{ng} / \mu 1 \mathrm{TGF}-\beta$ (Sigma-Aldrich; Merck KGaA) for 24,48 and $72 \mathrm{~h}$ at $37^{\circ} \mathrm{C}$. To determine the effect of miR-325-3p on epithelial-to-mesenchymal transition (EMT), HT-29 cells were treated with or without TGF- $\beta$ for $24 \mathrm{~h}$ at $37^{\circ} \mathrm{C}$. Subsequently, the cells were transfected with or without miR-325-3p inhibitors for $48 \mathrm{~h}$, as aforementioned. The cells were collected for further analysis.

Statistical analysis. Statistical analysis was performed using SPPS 20.0 software (IBM Corp.). Data are presented as the mean \pm standard deviation. Two-tailed unpaired Student's t-test or paired Student's t-test (for tumor vs. adjacent non-cancer tissues) was used to compare differences between two groups. One-way ANOVA followed by Tukey's post hoc test were used to compare difference between multiple groups. A receiver operating characteristic (ROC) curve was used to analyze the area under the curve (AUC) in the diagnosis of patients with $\mathrm{CRC}$. $\mathrm{P}<0.05$ was considered to indicate a statistically significant difference.

\section{Results}

Microarray assay. Tissue and serum samples were collected from patients with CRC and healthy individuals. A microarray 


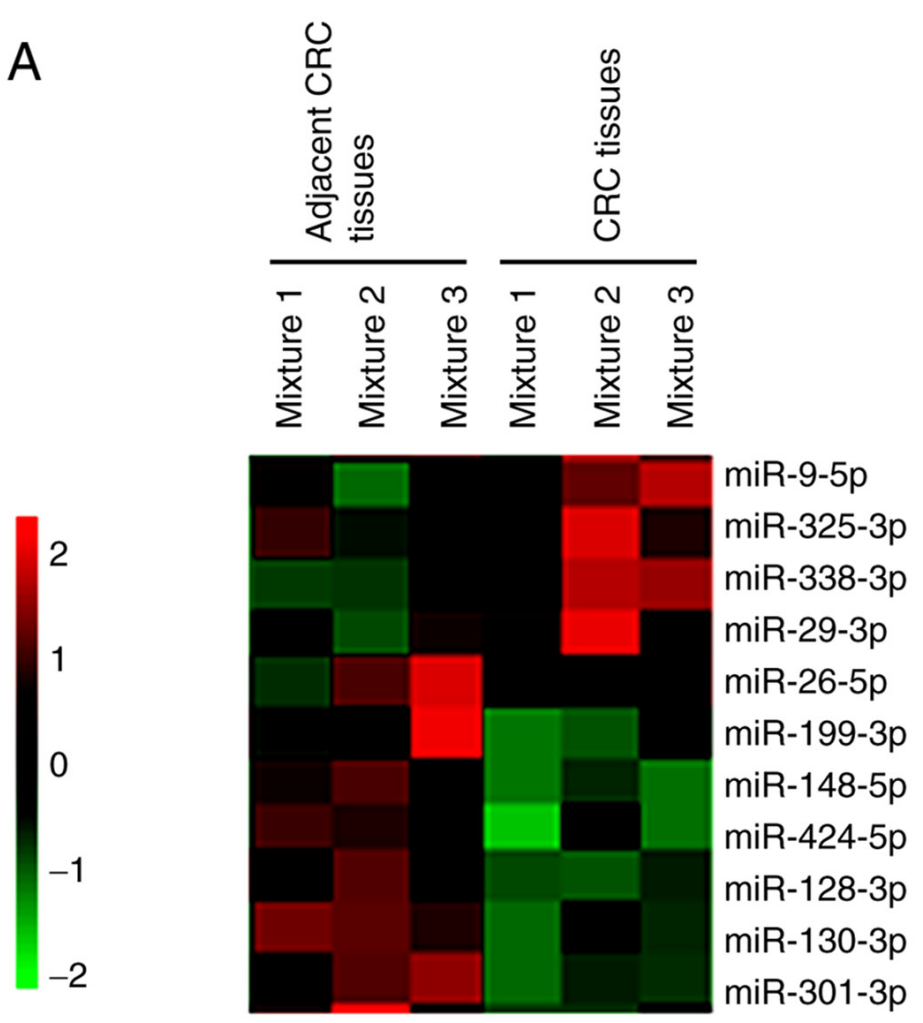

Tissue samples
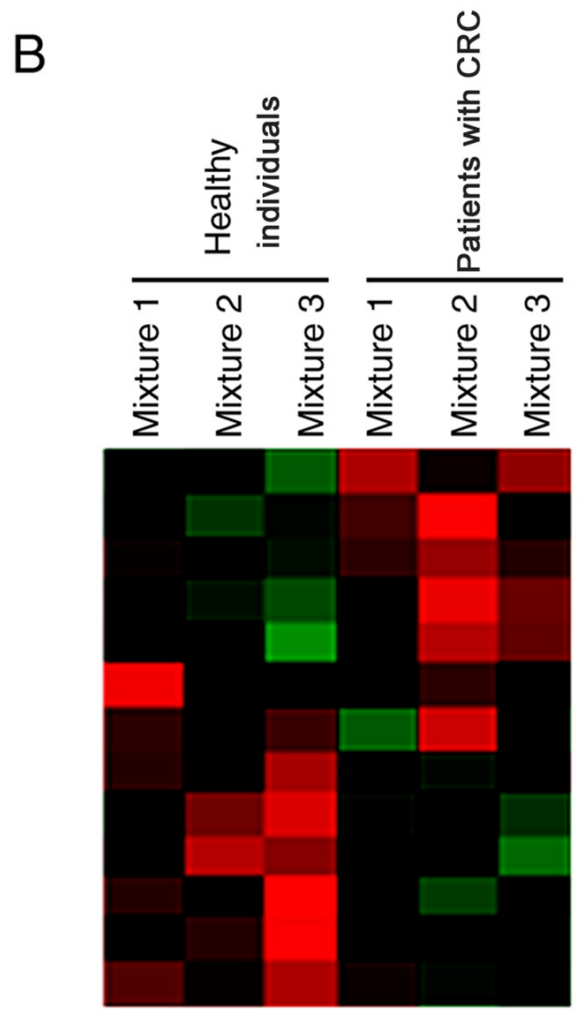

miR-18-5p

$\operatorname{miR}-98-5 p$

miR-325-3p

miR-142-5p

miR-497-5p

miR-19-3p

miR-18-5p

miR-152-3p

miR-222-3p

miR-311-3p

Serum samples

Figure 1. A microarray assay was performed to identify the differentially expressed miRNAs in the tissue and serum samples of patients with CRC and healthy individuals. miR-325-3p expression was significantly increased in both the (A) tissues and (B) serum samples of patients with CRC compared with the healthy individuals. miRNA/miR, microRNA; CRC, colorectal carcinoma.

assay was performed, and the results demonstrated that different miRNAs were dysregulated in the tissue and serum samples of patients with CRC and the healthy individuals. Notably, miR-325-3p expression was significantly upregulated in both the tissues and serum samples of patients with CRC compared with the healthy individuals (Fig. 1A and B).

miR-325-3p expression is elevated in patients with CRC. Based on the microarray data, RT-qPCR analysis was performed to detect miR-325-3p expression in patients with CRC. The results demonstrated that miR-325-3p expression was significantly upregulated in the tissues and serum samples of patients with CRC compared with the healthy individuals (Fig. 2A and B; $\mathrm{P}<0.001$ ).

Diagnostic value of miR-325-3p in patients with $C R C$. The ROC curve analysis was performed to determine the diagnostic value of tissue and serum miR-325-3p levels in patients with CRC. As presented in Fig. 3A, tissue miR-325-3p expression could be used to differentiate patients with CRC from healthy individuals, with an AUC value of 0.876 . When the cut-off value was 3.89 , the sensitivity was $95.4 \%$ and the specificity was $93.6 \%$. Furthermore, the AUC value of serum miR-325-3p expression was 0.795 in screening patients with CRC from healthy individuals. When the cut-off value was 11.5 , the sensitivity was $91.2 \%$ and the specificity was $87.6 \%$ (Fig. 3B). Taken together, these results suggest that miR-325-3p is a useful biomarker in identifying patients with CRC from healthy individuals.
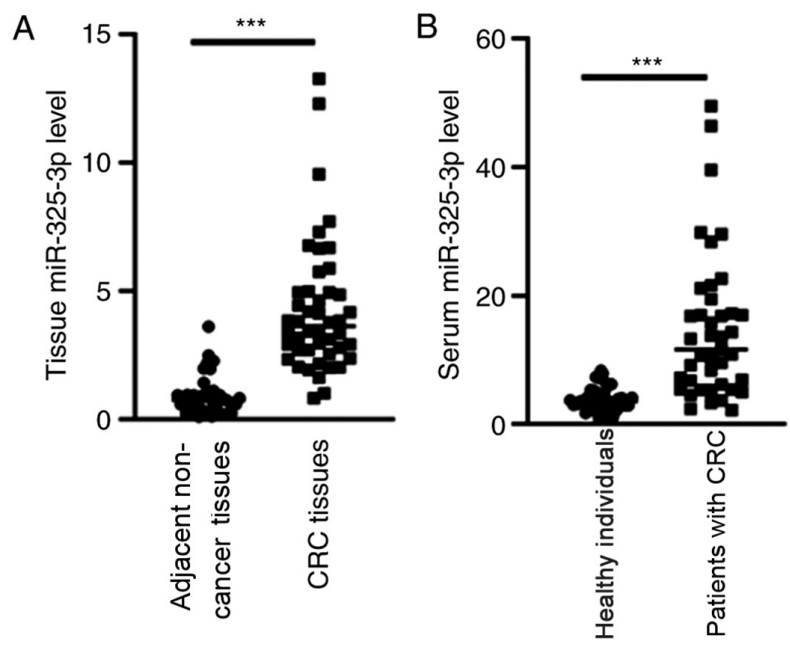

Figure 2. Tissue and serum miR-325-3p levels in patients with CRC and healthy individuals. miR-325-3p expression was significantly upregulated in the (A) tissues and (B) serum samples of patients with CRC compared with the healthy individuals. ${ }^{* * *} \mathrm{P}<0.001$. miR, microRNA; CRC, colorectal carcinoma.

Patients with CRC have higher serum miR-325-3p expression. The association between miR-325-3p expression and the clinicopathological characteristics of patients with CRC was assessed. As presented in Fig. 4A and D, tissue and serum miR-325-3p levels were significantly upregulated in patients 
A

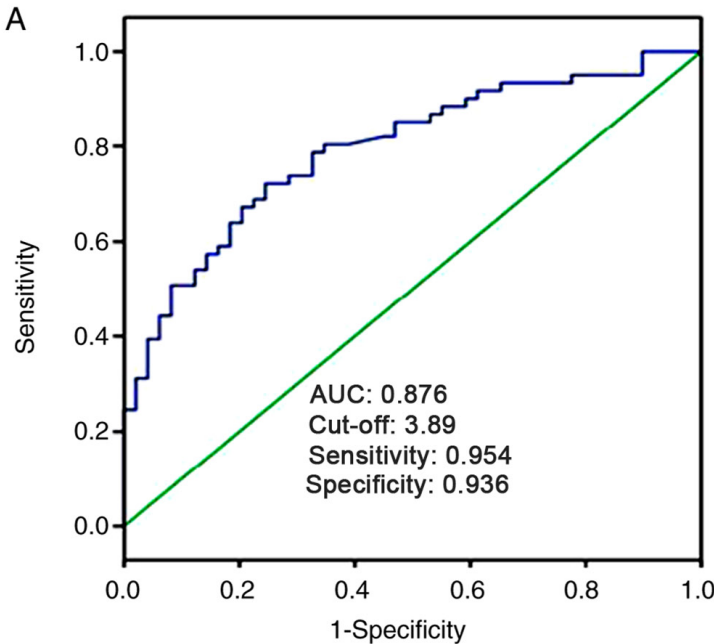

B

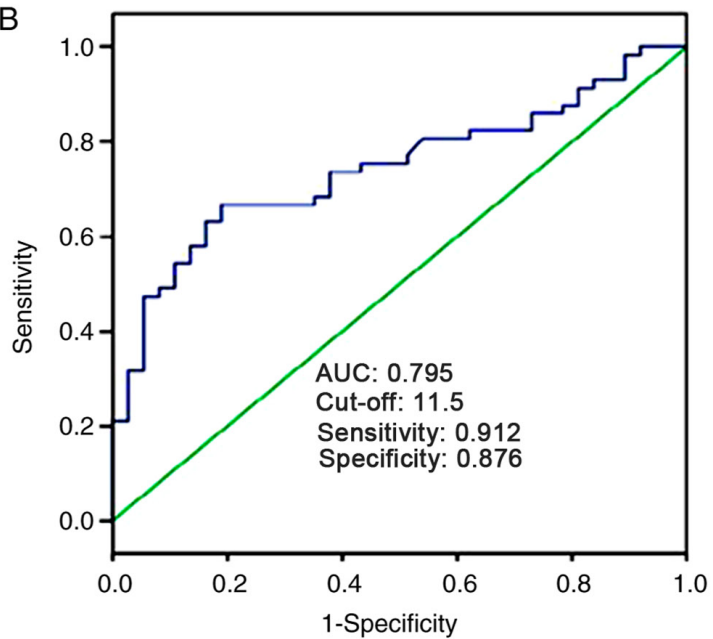

Figure 3. Receiver operating characteristic curve analysis was performed to determine the diagnostic value of tissue and serum miR-325-3p levels in patients with CRC. (A) Tissue miR-325-3p expression could be used to differentiate patients with CRC from healthy individuals, with an AUC value of 0.876. (B) The AUC value of serum miR-325-3p expression was 0.795 in screening patients with CRC from healthy individuals. miR, microRNA; AUC, area under the curve; CRC, colorectal carcinoma.

with well differentiated CRC than those with poorly differentiated CRC. In addition, tissue and serum miR-325-3p levels were significantly upregulated in patients at TNM stages III and IV compared with those at stages I and II (Fig. 4B and E). Furthermore, tissue and serum miR-325-3p levels were significantly upregulated in patients with lymph node metastasis (Fig. 4C and F).

miR-325-3p promotes the migration and invasion of $C R C$ cells. miR-325-3p expression was detected in the CRC cell lines, SW480, HCT116 and HT-29, as well as the normal colon cell line, FHC. The results demonstrated that miR-325-3p was highly expressed in HT-29 cells (Fig. 5A), thus, this cell line was used for subsequent experimentation. HT-29 cells were transfected with miR-325-3p mimics or inhibitors and RT-qPCR analysis was performed to assess transfection efficiency. The results demonstrated that transfection with miR-325-3p mimic significantly enhanced miR-325-3p expression, the effects of which were reversed following transfection with miR-325-3p inhibitor (Fig. 5B). The effect of miR-325-3p on the viability, migration and invasion of CRC cells was subsequently assessed. As presented in Fig. 5C, overexpression of miR-325-3p increased the cell viability in a time-dependent manner. In addition, the cell migratory and invasive abilities were significantly enhanced following transfection with miR-325-3p mimic (Fig. 5D). Conversely, miR-325-3p knockdown decreased HT-29 cell viability at 24, 48 and $72 \mathrm{~h}$ (Fig. 5E). Furthermore, the migratory and invasive abilities of HT-29 cells decreased following transfection with miR-325-3p inhibitors (Fig. 5F). Collectively, these results suggest that miR-325-3p acts as an oncogene in CRC.

CK18 is a target gene of miR-325-3p. Notably, a conserved binding of miR-325-3p was identified in the 3'-UTR of CK18 (Fig. 6A). The results of the dual-luciferase reporter assay demonstrated that miR-325-3p significantly suppressed the relative luciferase activity of the pmirGLO-CK18-3'-UTR compared with that of the blank pmirGLO plasmid (Fig. 6B). Western blot analysis demonstrated that overexpression of miR-325-3p significantly suppressed CK18 expression; however, miR-325-3p knockdown significantly elevated CK18 expression (Fig. 6C). Taken together, these results suggest that CK18 is a target gene of miR-325-3p.

miR-325-3p knockdown partially reverses transforming growth factor (TGF)- $\beta$-induced CK18 downregulation. The results demonstrated that treatment with TGF- $\beta$ upregulated miR-325-3p expression in a time-dependent manner (Fig. 7A). Conversely, TGF- $\beta$ significantly decreased CK18 expression at 48 and $72 \mathrm{~h}$ (Fig. 7B). Western blot analysis demonstrated that TGF- $\beta 1$ significantly decreased the expression of the epithelial marker, CK18, and increased the expression levels of the mesenchymal markers, $\alpha$-SMA and vimentin (Fig. 7C). Notably, these effects were reversed following inhibition of miR-325-3p (Fig. 7C). Collectively, these results suggest that miR-325-3p is a key regulator in TGF- $\beta$-induced CK18 downregulation.

\section{Discussion}

With the development of the economy, the incidence rate of CRC continues to increase and has become one of the most notable threats to human health (9). Despite advancements in endoscopic diagnosis and surgical techniques for CRC, the 5 -year survival rate was slightly $>10 \%$ in patients with stage IV disease $(18,19)$. The differential expression of several miRNAs in tumor tissues and normal tissues suggests that miRNAs may be used as molecular markers for tumor diagnosis $(7,8)$. As non-invasive markers, circulating miRNAs are expected to be sensitive and act as specific markers for tumor diagnosis and prognosis evaluation (20).

The present study screened miRNAs that are differentially expressed in the tissues and serum of patients with CRC. The results demonstrated that miR-325-3p tissue and serum levels were significantly upregulated in patients with 
A
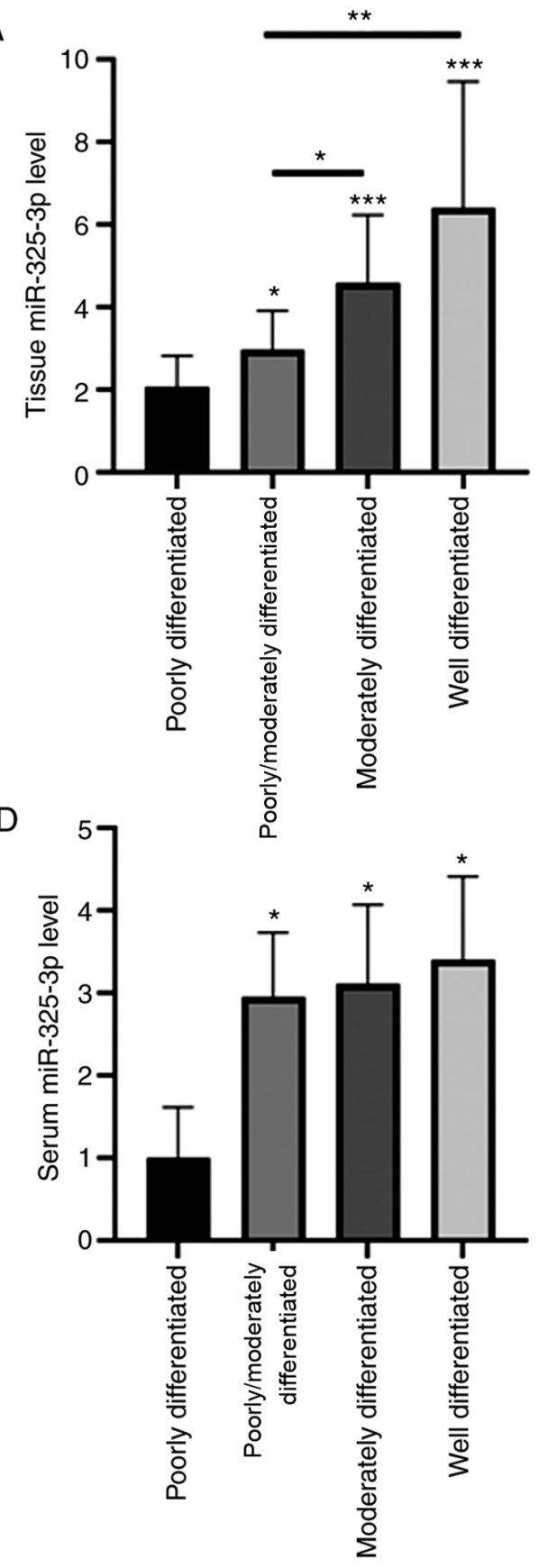

B

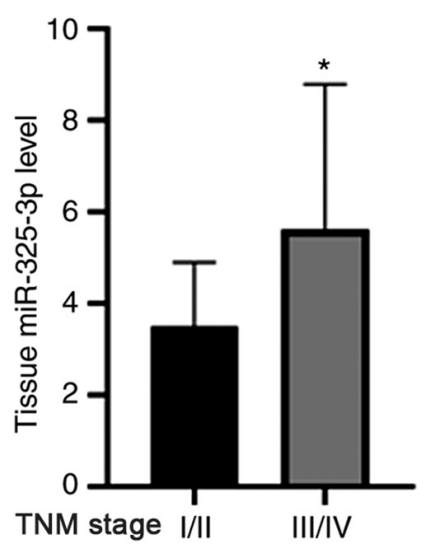

$\mathrm{E}$

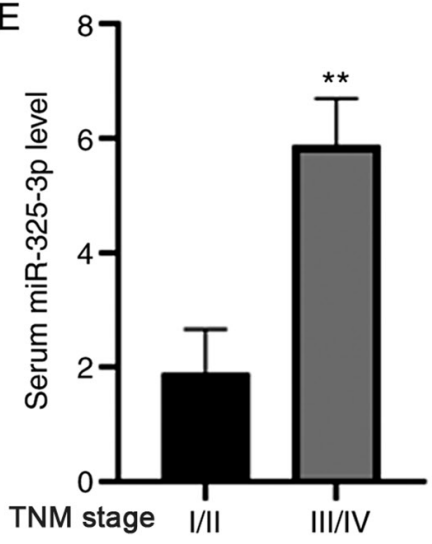

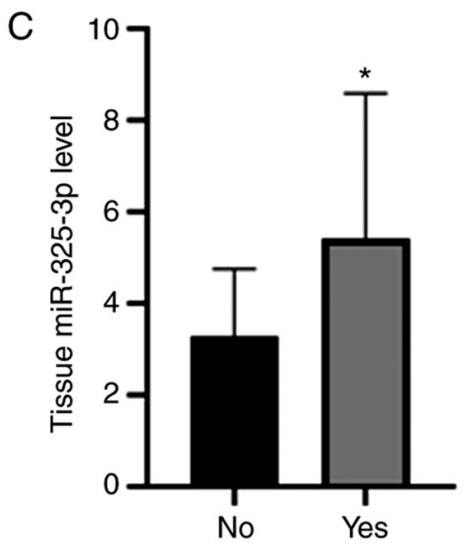

Lymph node metastasis
$\mathrm{F}$

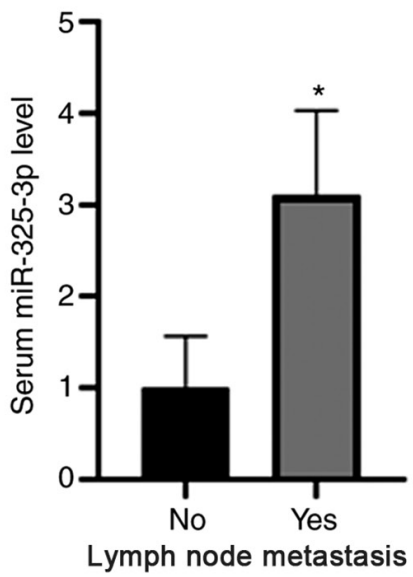

Figure 4. Tissue and serum miR-325-3p levels in patients with CRC. (A) Tissue miR-325-3p levels were significantly upregulated in patients with well differentiated CRC than those with poorly/moderately differentiated CRC. (B) miR-325-3p expression was also significantly upregulated in the tissue of patients at TNM stages III and IV than those at stages I and II. (C) miR-325-3p expression was also significantly upregulated in the tissue of patients with lymph node metastasis. (D) The level of serum miR-325-3p was determined in the serum of patients with CRC. (E) The level of serum miR-325-3p was increased in patients with CRC at stage III/IV compared with that in patients with stage I/II. (F) Elevated level of serum miR-325-3p was observed in patients with CRC with lymph node metastasis compared with that in patients without lymph node metastasis. ${ }^{*} \mathrm{P}<0.05 ;{ }^{* * *} \mathrm{P}<0.01 ;{ }^{* * * *} \mathrm{P}<0.001$ vs. poorly differentiated. miR, microRNA; $\mathrm{CRC}$, colorectal carcinoma; TNM, tumor-node-metastasis.

CRC compared with healthy individuals. In addition, ROC curve analyses demonstrated that both tissue and serum miR-325-3p levels may be used as indicators for the diagnosis of CRC, respectively. Furthermore, high miR-325-3p expression was significantly associated with tumor differentiation, TNM stage and lymph node metastasis. Taken together, these results suggest that miR-325-3p expression can be used as an indicator to monitor the progression of patients with CRC.
EMT is an important mechanism that affects the invasion and metastasis of CRC (21). Several growth factors, such as TGF- $\beta$ and epidermal growth factor, mediate EMT $(22,23)$. Notably, the results of the present study demonstrated that TGF- $\beta$ induced miR-325-3p expression. In addition, CK18 was identified as a target gene of miR-325-3p, suggesting that miR-325-3p is associated with the degree of EMT in CRC.EMT is widely involved in the invasion and metastasis of colon cancer due to the loss of epithelial characteristics into mesenchymal 

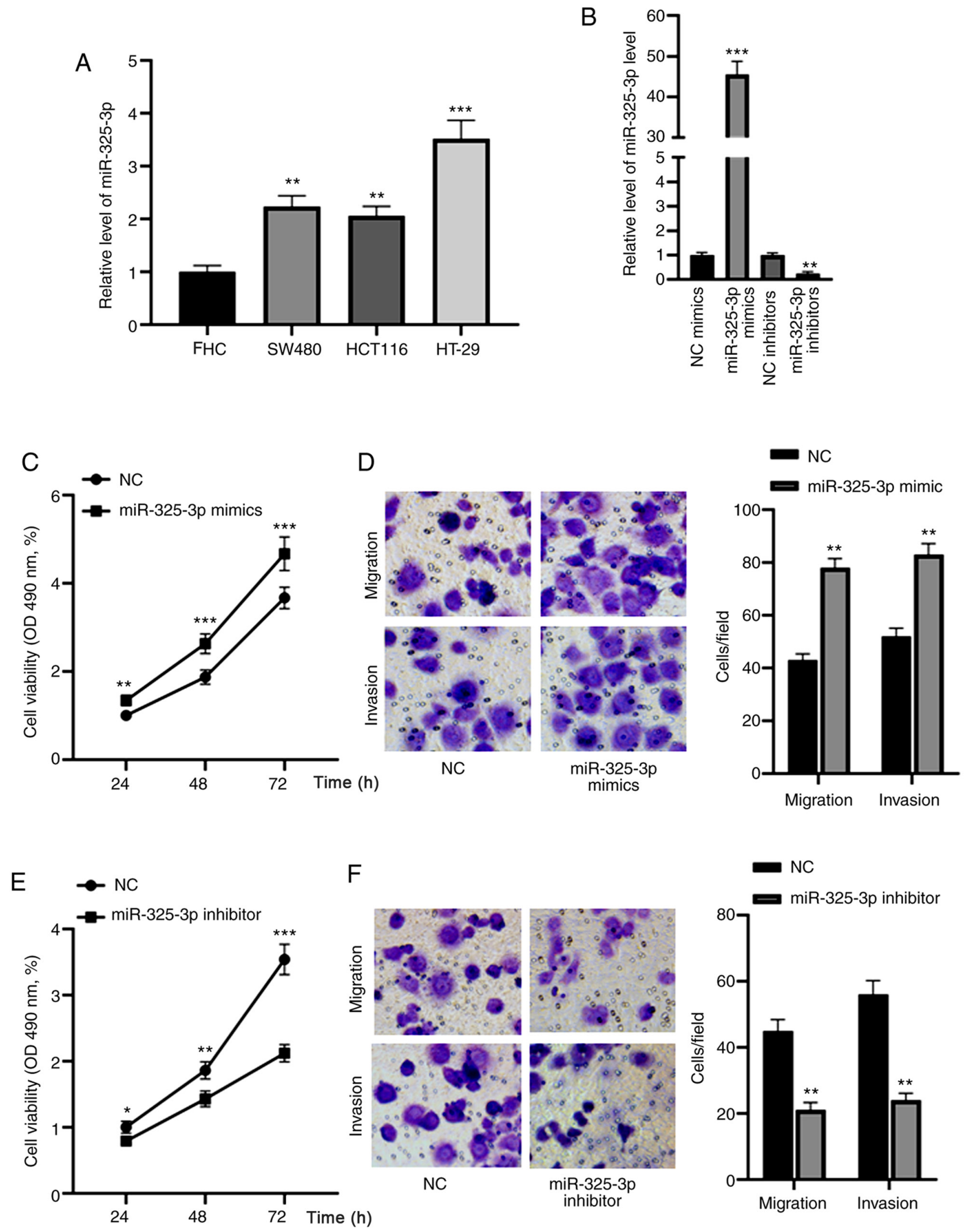

Figure 5. miR-325-3p promotes the migration and invasion of CRC cells. (A) RT-qPCR analysis was performed to detect miR-325-3p expression in the CRC cell lines, SW480, HCT116 and HT-29, as well as the normal colon cell line, FHC. (B) RT-qPCR analysis was performed to assess the transfection efficiency of miR-325-3p mimics or inhibitors into HT-29 cells. (C) The results of the MTT assay demonstrated that overexpression of miR-325-3p increased cell viability in a time-dependent manner. (D) Cell migratory and invasive abilities were significantly enhanced following transfection with miR-325-3p mimic. (E) miR-325-3p knockdown decreased HT-29 cell viability at 24, 48 and $72 \mathrm{~h}$. (F) The migratory and invasive abilities of HT-29 cells significantly decreased following transfection with miR-325-3p inhibitors. ${ }^{*} \mathrm{P}<0.05 ;{ }^{* * *} \mathrm{P}<0.01$; ${ }^{* * *} \mathrm{P}<0.001$. miR, microRNA; CRC, colorectal carcinoma; RT-qPCR, reverse transcription-quantitative PCR; NC, negative control; OD, optical density.

cells $(24,25)$. The results of the present study demonstrated that high miR-325-3p expression significantly decreased the expression of the epithelial marker, CK18, but increased the expression levels of the mesenchymal markers, vimentin and $\alpha$-SMA. It is suggested that HT-29 cells gradually lose the characteristics of epithelial cells and attain the characteristics of mesenchymal 
A

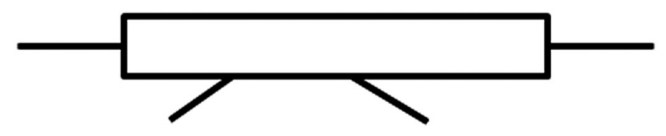

Position 102-108 of CK18 3'UTR CUUCUCCUGACCUGCCAAUAAAA

has-miR-325-3p

IIIIIII

AACUAUCCUCCAGGAGUUAUUU

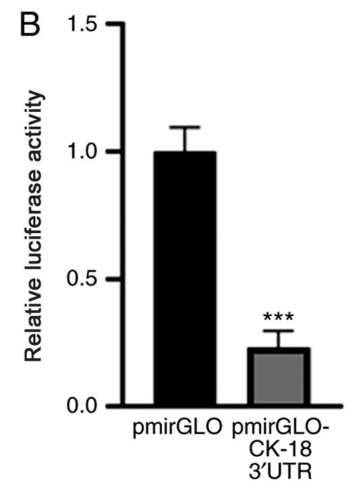

C
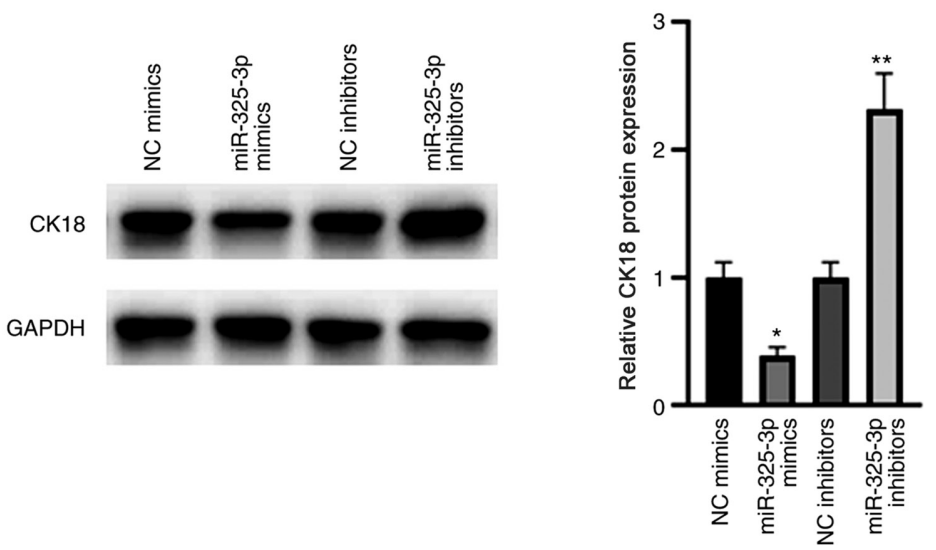

Figure 6. CK18 is a target gene of miR-325-3p. (A) Based on the TargetScan database, a conserved binding site of miR-325-3p was identified in the 3'-UTR of CK18. (B) The results of the dual-luciferase reporter assay demonstrated that miR-325-3p significantly suppressed the relative luciferase activity of pmirGLO-CK18-3'-UTR compared with that of the blank pmirGLO plasmid. (C) Western blot analysis demonstrated that overexpression of miR-325-3p significantly suppressed CK18 expression; however, miR-325-3p knockdown significantly elevated CK18 expression. ${ }^{*} \mathrm{P}<0.05 ;{ }^{* *} \mathrm{P}<0.01 ;{ }^{* * * *} \mathrm{P}<0.001$ vs. pmirGLO or NC mimics/inhibitors. CK18, cytokeratin 18; miR, microRNA; UTR, untranslated region; NC, negative control.

A

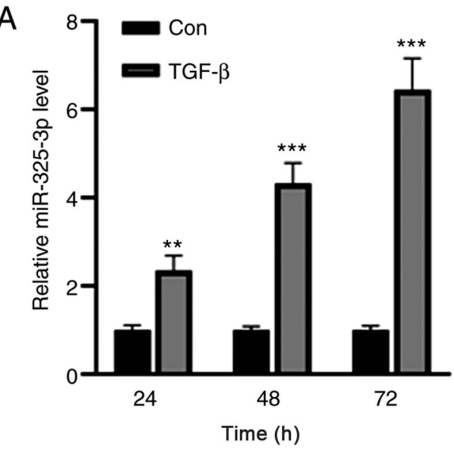

C

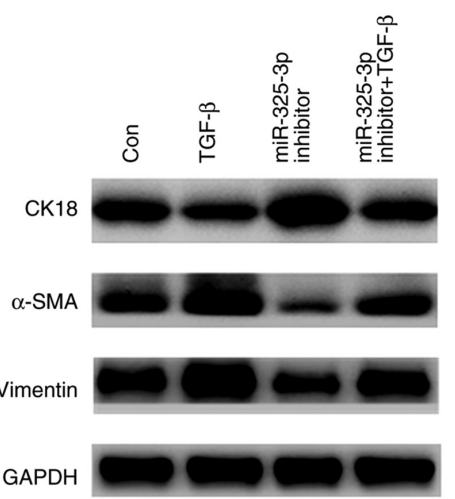

B

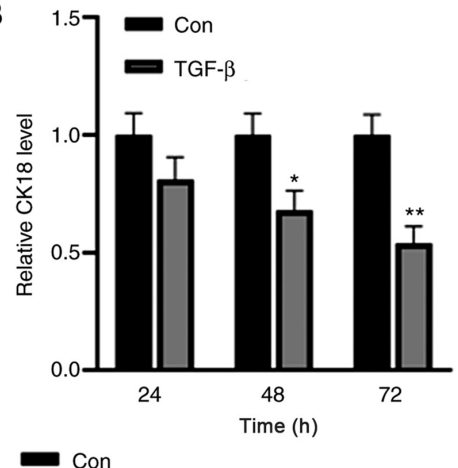

$\longrightarrow$ TGF- $\beta$

miR-325-3p inhibitor

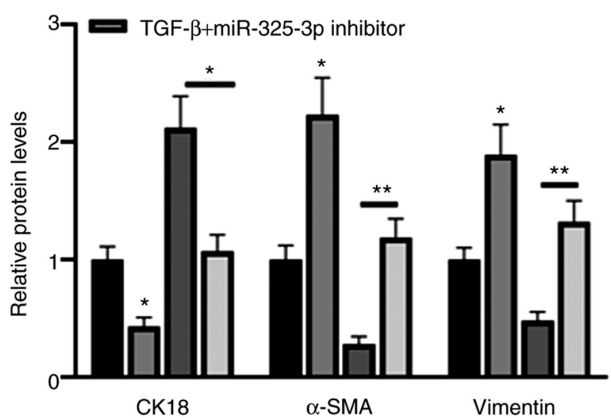

Figure 7. Inhibition of miR-325-3p partially reverses TGF- $\beta$-induced CK18 downregulation. (A) TGF- $\beta$ treatment upregulated miR-325-3p expression in a time-dependent manner. (B) TGF- $\beta$ treatment decreased CK18 expression at 48 and $72 \mathrm{~h}$. (C) TGF- $\beta 1$ significantly decreased the expression of the epithelial marker, CK18, and increased the expression levels of the mesenchymal markers, $\alpha$-SMA and vimentin, the effects of which were reversed following inhibition of miR-325-3p. ${ }^{*} \mathrm{P}<0.05 ;{ }^{* *} \mathrm{P}<0.01 ;{ }^{* * * *} \mathrm{P}<0.001$ vs. Con. miR, microRNA; TGF, transforming growth factor; CK18, cytokeratin 18 ; Con, control. 
cells, which are more likely to detach from the tumor and transfer to other areas of the body (26). Collectively, the results of the present study suggest that upregulated miR-325-3p expression in the tissue and serum samples of patients with CRC is closely associated with the progression of CRC.

The present study is not without limitations. First, the sample size was relatively small. Secondly, due to time constraints, the present study was unable to investigate the predictive value of serum miR-325-3p in patients with CRC.

In conclusion, elevated miR-325-3p expression is an important factor that affects EMT, and is likely to act as a molecular marker and potential therapeutic target in the progression of CRC. However, further studies are required to validate the results presented here.

\section{Acknowledgements}

Not applicable.

\section{Funding}

The present study was supported by a grant from the Hongqi Hospital Affiliated to Mudanjiang Medical University (grant no. 20190158).

\section{Availability of data and materials}

The datasets used and/or analyzed during the present study are available from the corresponding author upon reasonable request.

\section{Authors' contributions}

CS performed the experiments, analyzed the data and drafted the initial manuscript. XW, XZ, JA and YQ helped perform reverse transcription-quantitative $\mathrm{PCR}$ and western blot analyses. AC designed the experiments and analyzed the data. All authors have read and approved the final manuscript.

\section{Ethics approval and consent to participate}

The present study was approved by the Research Ethics Committee of Hongqi Hospital Affiliated to Mudanjiang Medical University (Mudanjiang, China; approval no. MDJHQ-20180925) and written informed consent was provided by all participants prior to the study start.

\section{Patient consent for publication}

Not applicable.

\section{Competing interests}

The authors declare that they have no competing interests.

\section{References}

1. Xu L, Zhang Y, Wang H, Zhang G, Ding Y and Zhao L: Tumor suppressor miR-1 restrains epithelial-mesenchymal transition and metastasis of colorectal carcinoma via the MAPK and PI3K/AKT pathway. J Transl Med 12: 244, 2014.
2. Zhang Y, Chen Z and Li J: The current status of treatment for colorectal cancer in China: A systematic review. Medicine (Baltimore) 96: e8242, 2017.

3. Xu Y, Han S, Lei K, Chang X, Wang K, Li Z and Liu J: Anti-Warburg effect of rosmarinic acid via miR-155 in colorectal carcinoma cells. Eur J Cancer Prev 25: 481-489, 2016.

4. Zhang H, Wang R and Wang M: miR-331-3p suppresses cell invasion and migration in colorectal carcinoma by directly targeting NRP2. Oncol Lett 18: 6501-6508, 2019.

5. Zhao DW, Li MM, Han JP, Wang Y, Jiang LX and Chang HL: miR-30c exerts tumor suppressive functions in colorectal carcinoma by directly targeting BCL9. Eur Rev Med Pharmacol Sci 23: 3335-3343, 2019.

6. Ding T, Cui P, Zhou Y, Chen C, Zhao J, Wang H, Guo M, $\mathrm{He} \mathrm{Z}$ and $\mathrm{Xu} \mathrm{L}$ : Antisense oligonucleotides against miR-21 inhibit the growth and metastasis of colorectal carcinoma via the DUSP8 pathway. Mol Ther Nucleic Acids 13: 244-255, 2018.

7. Fasihi A, M Soltani B, Atashi A and Nasiri S: Introduction of hsa-miR-103a and hsa-miR-1827 and hsa-miR-137 as new regulators of Wnt signaling pathway and their relation to colorectal carcinoma. J Cell Biochem 119: 5104-5117, 2018.

8. Ghanbari R, Mosakhani N, Asadi J, Nouraee N, Mowla SJ, Yazdani Y, Mohamadkhani A, Poustchi H, Knuutila S and Malekzadeh R: Downregulation of plasma MiR-142-3p and MiR-26a-5p in patients with colorectal carcinoma. Iran J Cancer Prev 8: e2329, 2015.

9. Gong L, Ren M, Lv Z, Yang Y and Wang Z: MiR-92b-3p promotes colorectal carcinoma cell proliferation, invasion, and migration by inhibiting FBXW7 in vitro and in vivo. DNA Cell Biol 37: 501-511, 2018

10. Guo H, Chen Y, Hu X, Qian G, Ge S and Zhang J: The regulation of Toll-like receptor 2 by miR-143 suppresses the invasion and migration of a subset of human colorectal carcinoma cells. Mol Cancer 12: 77, 2013.

11. Iacona JR and Lutz CS: MiR-146a-5p: Expression, regulation, and functions in cancer. Wiley Interdiscip Rev RNA 10: e1533, 2019.

12. Yang G, Xiong G, Cao Z, Zheng S, You L, Zhang T and Zhao Y: MiR-497 expression, function and clinical application in cancer. Oncotarget 7: 55900-55911, 2016.

13. Kulda V, Pesta M, Topolcan O, Liska V, Treska V, Sutnar A, Rupert K, Ludvikova M, Babuska V, Holubec L Jr and Cerny R: Relevance of miR-21 and miR-143 expression in tissue samples of colorectal carcinoma and its liver metastases. Cancer Genet Cytogenet 200: 154-160, 2010.

14. Kontos CK, Tsiakanikas P, Avgeris M, Papadopoulos IN and Scorilas A: MiR-15a-5p, a novel prognostic biomarker, predicting recurrent colorectal adenocarcinoma. Mol Diagn Ther 21: 453-464, 2017.

15. Livak KJ and Schmittgen TD: Analysis of relative gene expression data using real-time quantitative PCR and the 2(-Delta Delta C(T)) method. Methods 25: 402-408, 2001.

16. Guo J, Fang W, Sun L, Lu Y, Dou L, Huang X, Tang W, Yu L and Li J: Ultraconserved element uc.372 drives hepatic lipid accumulation by suppressing miR-195/miR4668 maturation. Nat Commun 9: 612, 2018

17. Yang F, Wang H, Jiang Z, Hu A, Chu L, Sun Y and Han J: MicroRNA19a mediates gastric carcinoma cell proliferation through the activation of nuclear factor- $\kappa \mathrm{B}$. Mol Med Rep 12: 5780-5786, 2015 .

18. Chen S, Dai Y, Zhang X, Jin D, Li X and Zhang Y: Increased miR-449a expression in colorectal carcinoma tissues is inversely correlated with serum carcinoembryonic antigen. Oncol Lett 7: 568-572, 2014.

19. Brenner H, Werner S and Chen H: Multitarget stool DNA testing for colorectal-cancer screening. N Engl J Med 371: 184-185, 2014.

20. Igder S, Mohammadiasl J and Mokarram P: Altered miR-21, miRNA-148a expression in relation to KRAS mutation status as indicator of adenoma-carcinoma transitional pattern in colorectal adenoma and carcinoma lesions. Biochem Genet 57: 767-780, 2019.

21. Pal I, Rajesh Y, Banik P, Dey G, Dey KK, Bharti R, Naskar D, Chakraborty S, Ghosh SK, Das SK, et al: Prevention of epithelial to mesenchymal transition in colorectal carcinoma by regulation of the E-cadherin-beta-catenin-vinculin axis. Cancer Lett 452: 254-263, 2019. 
22. Mylavarapu S, Kumar H, Kumari S, Sravanthi LS, Jain M, Basu A, Biswas M, Mylavarapu SVS, Das A and Roy M: Activation of epithelial-mesenchymal transition and altered $\beta$-catenin signaling in a novel Indian colorectal carcinoma cell line. Front Oncol 9: 54, 2019.

23. Niknami Z, Muhammadnejad A, Ebrahimi A, Harsani Z and Shirkoohi R: Significance of E-cadherin and Vimentin as epithelial-mesenchymal transition markers in colorectal carcinoma prognosis. EXCLI J 19: 917-926, 2020.

24. Wang JJ, Chong QY, Sun XB, You ML, Pandey V, Chen YJ, Zhuang QS, Liu DX, Ma L, Wu ZS, et al: Autocrine hGH stimulates oncogenicity, epithelial-mesenchymal transition and cancer stem cell-like behavior in human colorectal carcinoma. Oncotarget 8: 103900-103918, 2017.
25. Wang P, Gao XY, Yang SQ, Sun ZX, Dian LL, Qasim M, Phyo AT, Liang ZS and Sun YF: Jatrorrhizine inhibits colorectal carcinoma proliferation and metastasis through Wnt/ $\beta$-catenin signaling pathway and epithelial-mesenchymal transition. Drug Des Devel Ther 13: 2235-2247, 2019.

26. You S, Guan Y and Li W: Epithelialmesenchymal transition in colorectal carcinoma cells is mediated by DEK/IMP3. Mol Med Rep 17: 1065-1070, 2018.

(i) (3) This work is licensed under a Creative Commons Attribution-NonCommercial-NoDerivatives 4.0 International (CC BY-NC-ND 4.0) License. 